Proceedings

\title{
Membrane Proteins of Keratinocytes Protection by the Cannabidiol Applied before and after UVB Irradiation ${ }^{\dagger}$
}

\author{
Sinemyiz Atalay, Agnieszka Gęgotek and Elżbieta Skrzydlewska * \\ Department of Analytical Chemistry, Medical University of Białystok, 15-089 Białystok, Poland; \\ sinemyiz.atalay@umb.edu.pl (S.A.); agnieszka.gegotek@umb.edu.pl (A.G.) \\ * Correspondence: elzbieta.skrzydlewska@umb.edu.pl \\ + Presented at the 1st International e-Conference on Antioxidants in Health and Disease, 1-15 December \\ 2020; Available online: https://cahd2020.sciforum.net/.
}

Published: 30 November 2020

\begin{abstract}
The continuous increase in daily exposure to ultraviolet radiation, which influence on the redox state of skin cells, may contribute to the damage to the structure and function of cellular macromolecules, which favors the search for protective compounds. One promising compound is cannabidiol (CBD), phytocannabinoid which has antioxidant and anti-inflammatory properties. Therefore, the aim of this study was to compare the effect of CBD applied after (treatment) as well as before and after (pretreatment + treatment) keratinocytes irradiation with UVB on the proteomic profile of membrane proteins. Obtained data showed that both UVB radiation and CBD treatment significantly modified the proteomic profile of keratinocyte membranes. UVB was shown to dramatically increase the expression of proteins involved in the regulation of cell translation and proliferation (S3a/L13A/L7a ribosomal proteins), calcium ion homeostasis and inflammatory response (S100/S100-A6 proteins) and cellular redox state (peroxireoxin-1). Long action of CBD (pretreatment + treatment) was more effective in preventing changes caused by UVB, compared to the action of CBD used only after UVB irradiation. The strong activity of CBD applied before and after UVB irradiation suggests that this phytocannabinoid is effective in protecting skin cells against UVB-induced changes, in the keratinocyte proteome.
\end{abstract}

Keywords: cannabidiol; UVB; skin cells; keratinocytes; oxidative stress; membrane; proteomic analysis

\section{Introduction}

Ultraviolet radiation (UVR), as a harmful physical factor, can damage the skin by disturbing the intracellular redox balance and intensifying inflammation mainly in epidermal cells-keratinocytes [1]. Moreover, by inducing precancerous mutations, it may initiate carcinogenesis [2]. Along with them, our exposure to UV radiation increases day by day due to the activity of the natural sun source and additional artificial sources, as well as the average decrease in ozone content [3]. In order to prevent skin diseases caused by constant exposure to UV radiation, compounds with a protective and/or therapeutic effect are sought.

A promising antioxidant and anti-inflammatory compound is cannabidiol (CBD), which is a pharmacologically active but non-psychoactive phytocannabinoid [4]. Interactions between CBD and its molecular targets, including cannabinoid receptors and other components of the endocannabinoid system, brings the therapeutic properties of CBD which have been evaluated in cardiovascular, neurodegenerative, cancer, and metabolic diseases, which are usually accompanied by oxidative 
The 1st International Electronic Conference on Antioxidants in Health and Disease, 1-15 December 2020

stress and inflammation [4]. It has been shown that CBD can reduce the UV-induced level of superoxide anions and increase antioxidant capacity and consequently inhibits the lipid peroxidation process by inducing transcription by the nuclear factor associated with erythroid 2 (Nrf2) and by inhibiting the transcription of the nuclear factor kappa B (NF- $\kappa B$ ) [5,6]. In addition, it has already been observed that CBD is able to penetrate keratinocytes, accumulating in the cells membrane [5]. In this way, it protects keratinocytes, preventing changes in the composition of the cells membrane associated with damage caused by UVB, which include a reduction in the level of polyunsaturated fatty acids, an increase in the level of sialic acid and lipid peroxidation products (malondialdehyde and 8-isoprostanes). Consequently, CBD protects the integrity of cell membranes and prevents the release of lactate dehydrogenase into the environment [5].

Therefore, the main aim of this study was to analyze the effect of CBD, applied only after as well as before and after UV irradiation of keratinocytes, on the proteomic profile of the membrane of these cells.

\section{Experiment}

SDS-Page/nanoHPLC/QExactiveOrbiTrap analysis was performed for prepared membrane fractions obtained experimental keratinocytes cell culture groups which are control cells [CTR], cells treated with $4 \mu \mathrm{M}$ CBD during $48 \mathrm{~h}(\mathrm{~h})$ [CBD; $48 \mathrm{~h}$ ], cells treated with CBD during $24 \mathrm{~h}$ [CBD; $24 \mathrm{~h}$ ], cells exposed to UVB (312 nm) at $60 \mathrm{~mJ} / \mathrm{cm}^{2}$ [UVB], cells cultured for $24 \mathrm{~h}$ before and after UVB irradiation in medium containing $4 \mu \mathrm{M}$ CBD [CBD+UVB+CBD] and cells cultured for $24 \mathrm{~h}$ after UVB irradiation in medium containing $4 \mu \mathrm{M}$ CBD [UVB+CBD].

Samples containing $30 \mu \mathrm{g}$ of proteins, were separated using 10\% Tris-Glycine SDS-PAGE gels. Following in gel digestion (using trypsin), obtained final peptide mixtures were separated using nanoHPLC [analytical C18 column with $2 \mu \mathrm{m}$ particle size using using an Ultimate 3000 (Dionex, Idstein, Germany)]. The gradient started at $3 \mathrm{~min}$ and raised to $60 \%$ eluent B (90\% acetonitrile $+0.03 \%$ formic acid) for $40 \mathrm{~min}$ (at this time $40 \%$ eluent A was contained $5 \%$ acetonitrile $+0.1 \%$ formic acid). And, the eluted peptides from column were analysed using a Q Exactive HF mass spectrometer with an electrospray ionization source (ESI) (Thermo Fisher Scientific, Bremen, Germany). The data were acquired with the Xcalibur software (Thermo Fisher Scientific, Bremen, Germany). Whole comprehensive procedure of the analysis using liquid chromatography-tandem mass spectrometry (LC-MS/MS) for peptide identification have been described previously in Gegotek's study [7]. Generated raw data from LC-MS/MS were analysed using Proteome Discoverer 2.0 (Thermo Fisher Scientific, Bremen, Germany).

The effects of $\mathrm{CBD}$ pre-treatment [CBD+UVB+CBD] and $\mathrm{CBD}$ treatment [UVB+CBD] on membrane obtained keratinocytes exposed to UVB were analysed separately. The results from individual protein label-free quantification were normalized by Z-score and log-transformed Perseus (Perseus 1.6.5.0, https://maxquant.net/perseus/). Quality control and biostatistical analysis including one-way ANOVA, post-hoc analysis, principle component analysis (PCA), volcano plots and heat map were completed using Perseus and open-source software MetaboAnalyst 4.0 (http://www.metaboanalyst.ca). And, protein annotations were done using open-source PANTHER (http://pantherdb.org/) comparing with NCBI database (https://www.ncbi.nlm.nih.gov/) and opensource STRING (https://string-db.org/).

\section{Results and Discussion}

Keratinocytes are an effective physicochemical barrier against environmental stressors [8]. Their plasma membrane and the cell membranes of other cellular organelles because of their key components including lipids, proteins and sugars, as in other cell types, play a vital role in cell structure, organization and signaling [9]. Due to its high-energy characteristics, UVB radiation is mainly absorbed by the epidermis and may induce an inflammatory response, DNA damage, and even carcinogenesis [10]. Our results clearly showed that the keratinocyte membrane proteome 
The 1st International Electronic Conference on Antioxidants in Health and Disease, 1-15 December 2020

undergoes a critical change after UVB irradiation. On the other hand, CBD used after irradiation of cells, and especially both before and after UVB irradiation, can modulate these changes.

In this study, total 513 proteins were identified in membrane fractions of keratinocytes. According to statistical analysis, 70 proteins were found with significantly changed protein expression within experimental groups. And, the PCA demonstrated that CBD pre-treatment brought along clustering of experimental groups more in themselves (CBD pre-treatment: Component 1-45.2\%; Component 2-11.7\%; CBD treatment: (Component 1-37\%; Component 2$15.9 \%$, Figure 1). Volcano graphs comparing the control group and the group exposed to UVB radiation with the groups treated with $\mathrm{CBD}$, before and after or only after irradiation, and also between groups treated with CBD, before and after or only after UVB irradiation, show that the proteomes of the keratinocyte membranes differented significantly between groups (Figure 2). In addition, the primary split in the upper hierarchical dendrogram in the heat map showed that samples that are treated with CBD for $48 \mathrm{~h}$ [CBD $(48 \mathrm{~h})$ ] and that are treated with CBD before and after UVB irradiation [CBD + UVB + CBD] cluster closer to independently of samples exposed to UVB radiation.

Proteins whose expression changed significantly after UVB irradiation, as well as after the use of CBD, both as treatment as well as pretreatment and treatment, are mainly ribosomal proteins that participate in translation and proteosomal activity, proteins with catalytic activity, proteins involved in cell signaling, DNA/RNA binding proteins and protein structural proteins which are obtained from the membrane fractions including the plasma membrane and the membranes of cellular organelles. In addition, some of these proteins were found to be involved in the cellular response to UVB radiation, including regulation of cell redox status, inflammation, and apoptosis with a significant change after CBD treatment before and after UVB exposure (Table 1).

Table 1. Fold changes between cell groups for level of proteins that expressions were significant changed in membrane fractions obtained keratinocytes (control group [CTR], cell group treated with $4 \mu \mathrm{M}$ CBD during $48 \mathrm{~h}$ [CBD $48 \mathrm{~h}$ ], cell group treated with CBD during $24 \mathrm{~h}$ [CBD 24)], cell group exposed to UVB $(312 \mathrm{~nm})$ at $60 \mathrm{~mJ} / \mathrm{cm}^{2}$ [UVB], cell group cultured for $24 \mathrm{~h}$ before and after UVB irradiation in medium containing $4 \mu \mathrm{M}$ CBD [CBD+UVB+CBD] and cell group for $24 \mathrm{~h}$ after UVB irradiation in medium containing $4 \mu \mathrm{M}$ CBD [UVB+CBD]).

\begin{tabular}{ccccc}
\hline \multicolumn{3}{c}{ Fold Change } \\
\hline Protein ID & Protein Name & UVB/CTR & UVB+CBD/UVB & CBD+UVB+CBD/UVB \\
\hline A8K4W0 & $\begin{array}{c}\text { 40S ribosomal protein } \\
\text { S3a }\end{array}$ & 58.92 & 2.59 & $\mathbf{1 . 6 5}$ \\
\hline M0QYS1 & $\begin{array}{c}\text { 60S ribosomal protein } \\
\text { L13a }\end{array}$ & 14.16 & 5.46 & $\mathbf{4 . 3 5}$ \\
\hline P62424 & 60S ribosomal protein & 44.59 & 3.49 & $\mathbf{3 . 2 7}$ \\
\hline Q06830 & Peroxiredoxin-1 & 148.92 & 1.09 & $\mathbf{0 . 7 6}$ \\
\hline V9HWH9 & Protein S100 & 1.68 & 1.84 & $\mathbf{0 . 0 2}$ \\
\hline P06703 & Protein S100-A6 & 40.81 & 3.31 & $\mathbf{1 . 5 3}$ \\
\hline
\end{tabular}

In this study, it was found that UVB radiation induces level of ribosomal proteins such as $40 \mathrm{~S}$ ribosomal protein S3a, 60S ribosomal proteins L13a and L7a, which are likely derived from the nuclear membrane or extracellular exosomes and which are involved in ribosomal complex formation, translation and NF- $\kappa$ B signaling. It has been shown that overexpression of the S3a ribosomal protein prevents drug-induced apoptosis and promotes neoplastic transformation [11]. On the other hand, increased expression of the L13a protein as well as L7a can induce apoptosis due to their participation in the cell cycle and translation through the accumulation of p53 [12]. Along with the induction, by CBD application, of the S3a protein level in UVB exposed membranes, CBD also induces levels of other pro-apoptotic ribosomal proteins. The tendency of a cell to apoptosis or to 
The 1st International Electronic Conference on Antioxidants in Health and Disease, 1-15 December 2020 differentiate is highly dependent on the metabolic environment and the specificity of the cells. Even if it is not possible to interpret one of these directions on the basis of these data alone, this situation may indicate that CBD, through its pro-apoptotic effects, protects cells from neoplastic transformation.
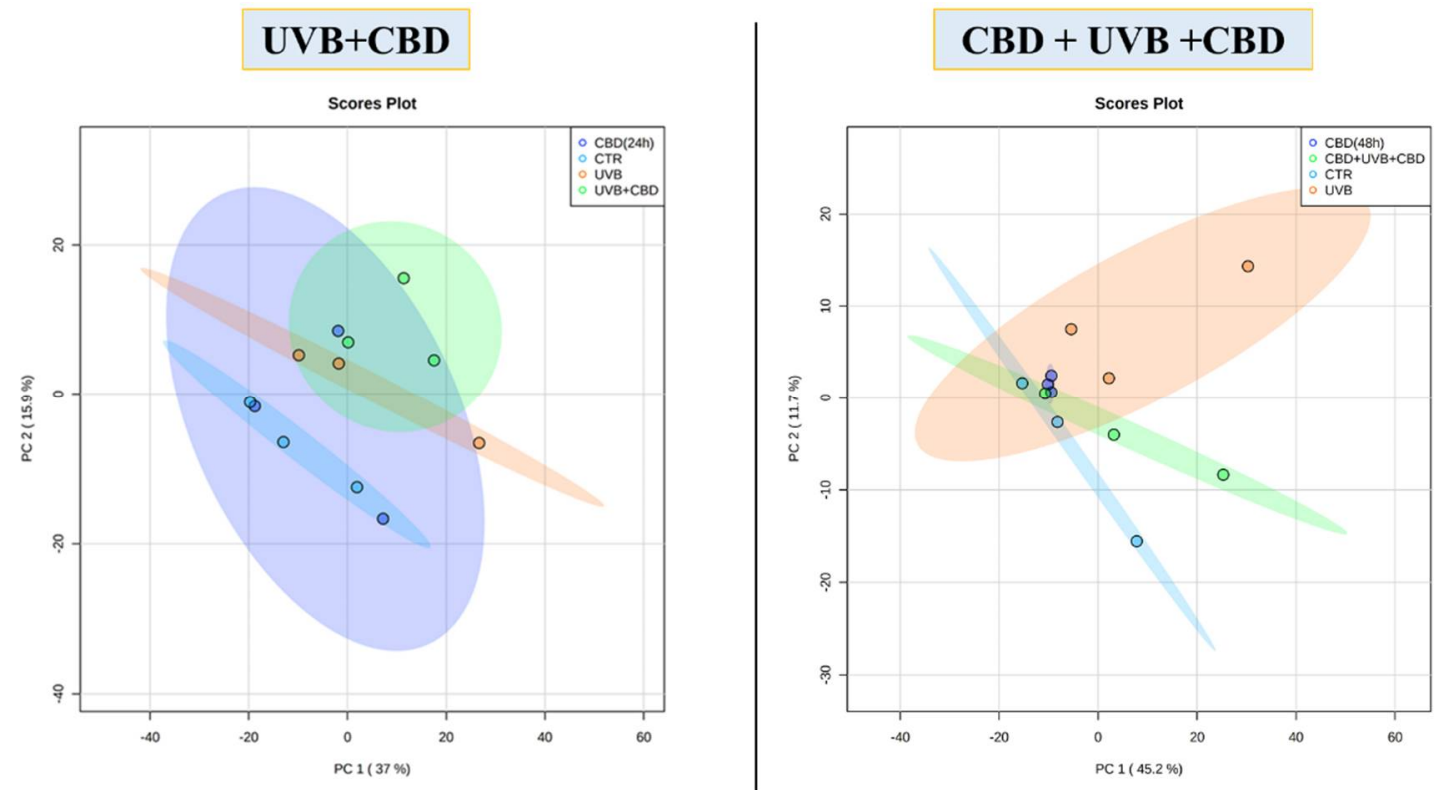

Figure 1. Principal component analysis (PCA) of membrane fractions obtained keratinocytes from control group [CTR], cell group treated with $4 \mu \mathrm{M} \mathrm{CBD}$ during $48 \mathrm{~h}$ [CBD $48 \mathrm{~h}$ ], cell group treated with CBD during $24 \mathrm{~h}$ [CBD $24 \mathrm{~h}$ ], cell group exposed to UVB $(312 \mathrm{~nm})$ at $60 \mathrm{~mJ} / \mathrm{cm}^{2}$ [UVB], cell group cultured for $24 \mathrm{~h}$ before and after UVB irradiation in medium containing $4 \mu \mathrm{M}$ CBD [CBD+UVB+CBD] and cell group for 24 hafter UVB irradiation in medium containing $4 \mu \mathrm{M} C B D$ [UVB+CBD].

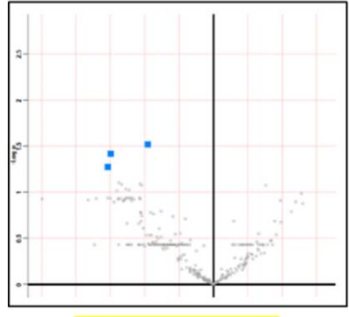

CBD (48h) - CTR

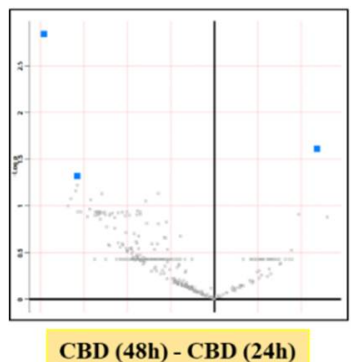

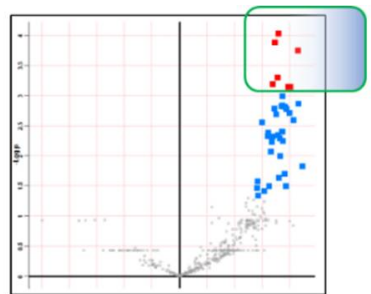

UVB - CTR

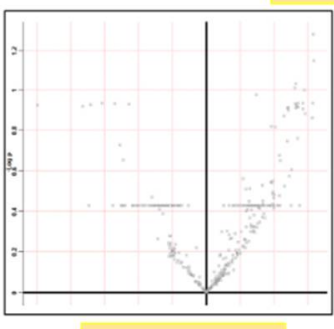

CBD (24h) - CTR
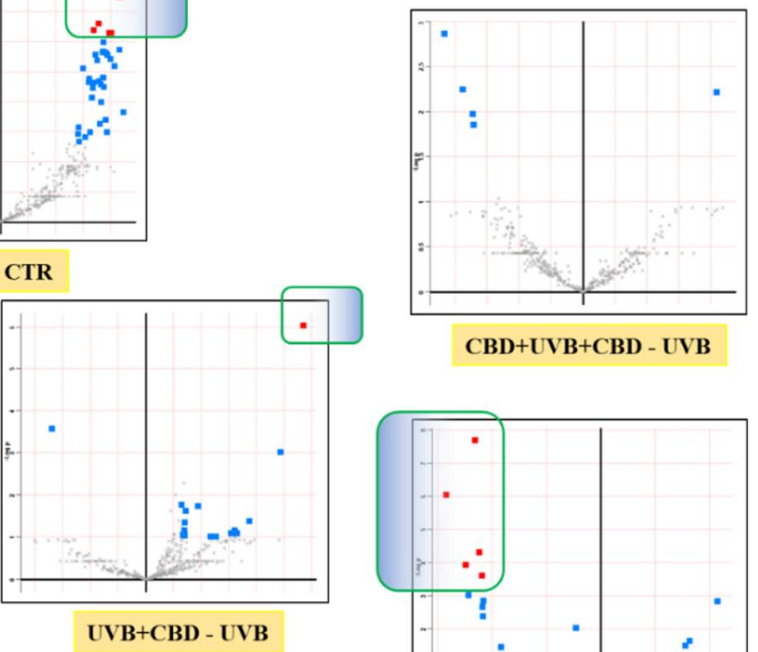

CBD+UVB+CBD - UVB

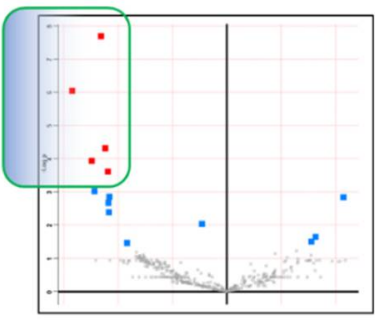

$\mathrm{CBD}+\mathrm{UVB}+\mathrm{CBD}-\mathrm{UVB}+\mathrm{CBD}$

Figure 2. Volcano plots comparing the effects of cannabidiol treatments ( $4 \mu \mathrm{M} \mathrm{CBD}$ for $48 / 24 \mathrm{~h} ; 4 \mu \mathrm{M}$ CBD for $24 \mathrm{~h}$ before and $24 \mathrm{~h}$ after UVB irradiation, $4 \mu \mathrm{M}$ CBD for $24 \mathrm{~h}$ after UVB irradiation) on keratinocyte membranes from control and UVB $\left(312 \mathrm{~nm}\right.$, at $\left.60 \mathrm{~mJ} / \mathrm{cm}^{2}\right)$ irradiated groups. (Significant features (in blue) had $p<0.05$; significant features (in red) had FDR-adjusted $p$-value $<0.05$ ). 
The 1st International Electronic Conference on Antioxidants in Health and Disease, 1-15 December 2020

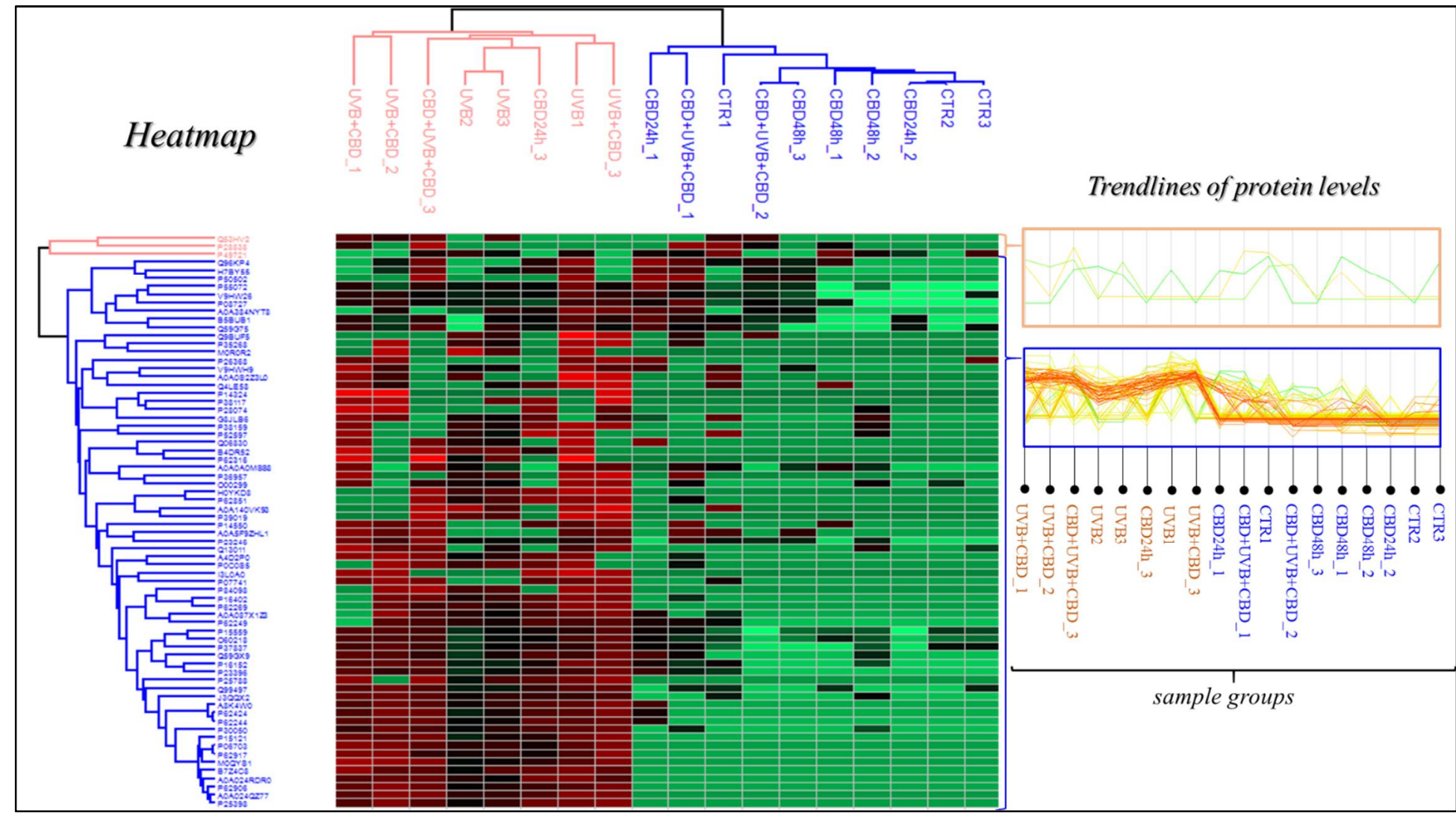

Figure 3. Heat map and clustering for significant proteins from control group [CTR], cell group treated with $4 \mu \mathrm{M} \mathrm{CBD}$ during $48 \mathrm{~h}$ [CBD $48 \mathrm{~h}$ ], cell group treated with CBD during $24 \mathrm{~h}$ [CBD $24 \mathrm{~h}$ ], cell group exposed to UVB $(312 \mathrm{~nm})$ at $60 \mathrm{~mJ} / \mathrm{cm}^{2}$ [UVB], cell group cultured for $24 \mathrm{~h}$ before and after UVB irradiation in medium containing $4 \mu \mathrm{M}$ CBD [CBD+UVB+CBD] and cell group for $24 \mathrm{~h}$ after UVB irradiation in medium containing $4 \mu \mathrm{M} \mathrm{CBD}$ [UVB+CBD]. 
The 1st International Electronic Conference on Antioxidants in Health and Disease, 1-15 December 2020

In addition, we have shown that the use of CBD after irradiation of keratinocytes with UVB radiation slightly increased the level of peroxiredoxin-1, a member of the antioxidant proteins, while the use of CBD both before and after irradiation with UVB cells reduced UVB-induced overexpression. In addition to its antioxidant effect, peroxiredoxin-1 also has anti-inflammatory properties through interaction with the DNA repair protein APE1, which may affect the DNA binding activity of NF- $\kappa B$ [13]. However, it is known that inhibition of APE1 activity also increases the level of Nrf2 target genes. Although this antioxidant and anti-inflammatory response from keratinocytes to UVB radiation may be induced by the relatively short duration of CBD's action in the cellular response to UVB-induced stress. On the other hand, longer exposure to CBD can counteract these changes. A similar trend to the reduction of antioxidant protein expression caused by the action of $\mathrm{CBD}$ has been recently demonstrated in the case of superoxide dismutase in the keratinocytes of rats exposed to UVA/B radiation [14].

We also observed that UVB radiation increased the level of the S100 protein, especially the S100A6 protein, which participates in calcium-dependent binding of proteins and the regulation of cell proliferation and apoptosis, as well as the cellular response to various stress factors due to the specificity of the cell type [15]. S100-A6 can induce or reduce apoptosis in several cell types [15]. On the other hand, it was shown that NF- $\kappa B$ and Nrf2 at the transcription level can activate the promoter of the S100A6 gene [15]. Thus, the increased level of S100-A6 caused by CBD treatment may be the result of a relationship between activation of the NF- $\kappa$ B promoter or Nrf2 and S100-A6. Moreover, along with its tendency to lower peroxiredoxin-1 level, this situation may also support the long-term protective effect of CBD against cell proliferation in the tumorigenesis through its pro-apoptotic effect, which is exacerbated by the level of pro-apoptotic factors such as the 605 ribosomal protein L7a [16]. However, since the use of CBD increased the level of the S100 protein as well as S100-A6 located in the nuclear membranes, the plasma membrane, or the extracellular exosome, CBD longaction increased this UVB-induced S100-A6 level, but it was 2 times lower than after CBD treatment and even CBD pre-treatment reduced the level of the S100 protein increased by UVB.

\section{Conclusions}

Our study showed that UVB radiation significantly modifies the proteome of keratinocytes membrane. The use of CBD can effectively modulate changes induced by UVB, mainly related to cell proliferation as well as oxidative and inflammatory state. Our data also show that the use of CBD both before and after irradiation of keratynocytes with UVB may be more effective in counteracting changes in membrane proteome. The protective effect of CBD against stress induced by UVB may result from the induction of pro-apoptotic and ribosomal proteins. This effect may also be the result of CBD interference in the crosstalk between NF- $\kappa B$ and Nrf2.

Acknowledgments: This research was conducted within the project which has received funding from the European Union's Horizon 2020 research and innovation programme under the Marie Skłodowska-Curie grant agreement No 754432 (and the Polish Ministry of Science and Higher Education, from financial resources for science in 2018-2023 granted for the implementation of an international co-financed project). SA is supported by above project.

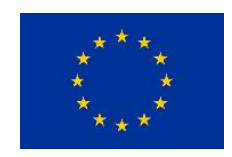

Conflicts of Interest: The authors declare no conflict of interest.

\section{References}

1. Panich, U.; Sittithumcharee, G.; Rathviboon, N.; Jirawatnotai, S. Ultraviolet radiation-induced skin aging: The role of DNA damage and oxidative stress in epidermal stem cell damage mediated skin aging. Stem Cells Int. 2016, 2016, 7370642, doi:10.1155/2016/7370642. 
The 1st International Electronic Conference on Antioxidants in Health and Disease, 1-15 December 2020

2. de Pedro, I.; Alonso-Lecue, P.; Sanz-Góme, N.; Freije, A.; Gandarillas, A. Sublethal UV irradiation induces squamous differentiation via a p53-independent, DNA damage-mitosis checkpoint. Cell Death Dis. 2018, 9 , 1094, doi:10.1038/s41419-018-1130-8.

3. Schuch, A.P.; Moreno, N.C.; Schuch, N.J.; Menck, C.F.M.; Garcia, C.C.M. Sunlight damage to cellular DNA: Focus on oxidatively generated lesions. Free Radic. Biol. Med. 2017, 107, 110-124, doi:10.1016/j.freeradbiomed.2017.01.029.

4. Atalay, S.; Jarocka-Karpowicz, I.; Skrzydlewska, E. Antioxidative and Anti-Inflammatory Properties of Cannabidiol. Antioxidants 2020, 9, 21, doi:10.3390/antiox9010021.

5. Atalay, S.; Dobrzyńska, I.; Gęgotek, A.; Skrzydlewska, E. Cannabidiol protects keratinocyte cell membranes following exposure to UVB and hydrogen peroxide. Redox Biol. 2020, 36, 101613, doi:10.1016/j.redox.2020.101613.

6. Jastrząb, A.; Gęgotek, A.; Skrzydlewska, E. Cannabidiol regulates the expression of keratinocyte proteins involved in the inflammation process through transcriptional regulation. Cells 2019, 8, 827, doi:10.3390/cells8080827.

7. Gęgotek, A.; Domingues, P.; Wroński, A.; Wójcik, P.; Skrzydlewska, E. Proteomic plasma profile of psoriatic patients. J. Pharm. Biomed. Anal. 2018, 155, 185-193, doi:10.1016/j.jpba.2018.03.068.

8. Parrado, C.; Mercado-Saenz, S.; Perez-Davo, A.; Gilaberte, Y.; Gonzalez, S.; Juarranz, A. Environmental Stressors on Skin Aging. Mechanistic Insights. Front. Pharmacol. 2019, 10, 759, doi:10.3389/fphar.2019.00759.

9. Watson, H.; Biological membranes. Essays Biochem. 2015, 59, 43-69, doi:10.1042/bse0590043.

10. D'Orazio, J.; Jarrett, S.; Amaro-Ortiz, A.; Scott, T. UV radiation and the skin. Int. J. Mol. Sci. 2013, 14, 1222212248, doi:10.3390/ijms140612222.

11. Zhou, X.; Liao, W.J.; Liao, J.M.; Liao, P.; Lu, H. Ribosomal proteins: Functions beyond the ribosome. J. Mol. Cell Biol. 2015, 7, 92-104, doi:10.1093/jmcb/mjv014.

12. Chen, F.W.; Ioannou, Y.A. Ribosomal proteins in cell proliferation and apoptosis. Int. Rev. Immunol. 1999, 18, 429-448, doi:10.3109/08830189909088492.

13. Nassour, H.; Wang, Z.; Saad, A.; Papaluca, A.; Brosseau, N.; Affar, E.B., Alaoui-Jamali, M.A.; Dindial, R. Peroxiredoxin 1 interacts with and blocks the redox factor APE1 from activating interleukin-8 expression. Sci. Rep. 2016, 6, 29389, doi:10.1038/srep29389.

14. Atalay, S.; Gęgotek, A.; Wroński, A.; Domigues, P.; Skrzydlewska, E. Therapeutic application of cannabidiol on UVA and UVB irradiated rat skin. A proteomic study. J. Pharm. Biomed. Anal. 2021, 192, 113656, doi:10.1016/j.jpba.2020.113656.

15. Donato, R.; Sorci, G.; Giambanco, I. S100A6 protein: Functional roles. Cell Mol. Life Sci. 2017, 74, 2749-2760, doi:10.1007/s00018-017-2526-9.

16. Xu, X.; Xiong, X.; Sun, Y. The role of ribosomal proteins in the regulation of cell proliferation, tumorigenesis, and genomic integrity. Sci. China Life Sci. 2016, 59, 656-672, doi:10.1007/s11427-016-0018-0.

Publisher's Note: MDPI stays neutral with regard to jurisdictional claims in published maps and institutional affiliations.

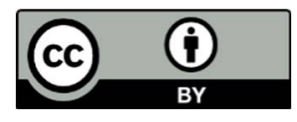

(C) 2020 by the authors; licensee MDPI, Basel, Switzerland. This article is an open access article distributed under the terms and conditions of the Creative Commons by Attribution (CC-BY) license (http://creativecommons.org/licenses/by/4.0/). 